\title{
Age dependency of central and peripheral systolic blood pressures: Cross-sectional and longitudinal observations in European populations
}

\author{
WIKTORIA WOJCIECHOWSKA ${ }^{1}$, KATARZYNA STOLARZ-SKRZYPEK ${ }^{1}$, \\ VALÉRIE TIKHONOFF ${ }^{2}$, TOM RICHART ${ }^{3,4}$, JITKA SEIDLEROVÁ ${ }^{5}$, MARCIN CWYNAR ${ }^{6}$, \\ LUTGARDE THIJS ${ }^{3}$, YAN LI ${ }^{7}$, TATIANA KUZNETSOVA ${ }^{3}$, JAN FILIPOVSKÝ ${ }^{5}$, \\ EDOARDO CASIGLIA ${ }^{2}$, TOMASZ GRODZICKI ${ }^{6}$, KALINA KAWECKA-JASZCZ $^{1}$, \\ MICHAEL O'ROURKE ${ }^{8}$, JAN A. STAESSEN ${ }^{3,4} \&$ ON BEHALF OF THE EUROPEAN \\ PROJECT ON GENES IN HYPERTENSION (EPOGH) INVESTIGATORS
}

\author{
${ }^{1}$ The First Department of Cardiology and Hypertension, Fagiellonian University Medical College, Kraków, Poland; \\ ${ }^{2}$ Department of Clinical and Experimental Medicine, University of Padova, Padova, Italy; ${ }^{3}$ Studies Coordinating Centre, \\ Division of Hypertension and Cardiovascular Rehabilitation, Department of Cardiovascular Diseases, University of \\ Leuven, Leuven, Belgium; ${ }^{4}$ Department of Epidemiology, Maastricht University, Maastricht, The Netherlands; \\ ${ }^{5}$ Faculty of Medicine in Pilsen, Charles University, Pilsen, the Czech Republic; ${ }^{6}$ Department of Internal Medicine \\ and Gerontology Medical College, Fagiellonian University, Kraków, Poland; ${ }^{7}$ Center for Epidemiologic Studies \\ and Clinical Trials and the Center for Vascular Evaluation, Shanghai Key Laboratory of Vascular Biology; \\ ${ }^{8}$ Saint Vincent's Clinic, University of New South Wales, Sidney, Australia
}

\begin{abstract}
Background. As arteries become stiffer with ageing, reflected waves move faster and augment late systolic pressure. We investigated the age dependency of peripheral and central systolic pressure, pressure amplification (peripheral systolic blood pressure - central systolic blood pressure), and peripheral and central systolic augmentation (maximal systolic pressure minus the first peak of the pressure wave). Methods. We randomly recruited 1420 White Europeans (mean age, 41.7 years). peripheral systolic blood pressure and central systolic blood pressure were measured by means of an oscillometric sphygmomanometer and pulse wave analysis, respectively. Results. In cross-sectional analyses (731 women, 689 men), central systolic blood pressure and central systolic augmentation increased more with age than peripheral systolic blood pressure and peripheral systolic augmentation. These age-related increases were greater in women than men. The age-related decrease in pressure amplification was similar in both sexes. In longitudinal analyses (208 women, 190 men), the annual increases in central systolic blood pressure and central systolic augmentation were steeper $(p<0.001)$ than those in peripheral systolic blood pressure and peripheral systolic augmentation with no sex differences $(p \geq 0.068)$, except for peripheral systolic augmentation, which was larger in women $(p=0.002)$. Longitudinally, pressure amplification decreased more with age in women than men $(p=0.012)$. In multivariable-adjusted analyses, age was the overriding determinant of peripheral systolic blood pressure and central systolic blood pressure. Conclusion. With ageing, peripheral systolic blood pressure approximates to central systolic blood pressure. This might explain why in older subjects peripheral systolic blood pressure becomes the main predictor of cardiovascular complications.
\end{abstract}

Key Words: ageing, cardiovascular disease, central blood pressure, epidemiology, peripheral blood pressure, risk factors

\section{Introduction}

Systolic blood pressure substantially rises with ageing across the full human life span (1). However, because arteries stiffen with higher age and because of differences in wave travel distance and wave reflections, the age-related increase in systolic blood pressure does not occur uniformly across the whole arterial tree (2). Indeed, during systole, the heart

Correspondence: Jan A. Staessen, Studies Coordinating Centre, Laboratory of Hypertension, Campus Sint Rafaël, Kapucijnenvoer 35, Block D, Box 7001, BE-3000 Leuven, Belgium. Tel: +32-16-34-7104. Fax:+32-16-34-7106. E-mail: jan.staessen@med.kuleuven.be 
generates a forward running pressure wave, which is reflected at various sites in the peripheral arterial system (2). In healthy young adults, the reflected waves coincide with diastole and raise diastolic pressure. As the arteries become stiffer with advancing age, the reflected waves move faster, reach the proximal aorta during systole and cause an augmentation of late systolic pressure, whereas diastolic pressure decreases.

Augmentation of systolic blood pressure in the central arteries increases with advancing age. Because of this phenomenon, there might be differences in the age dependency of peripheral systolic blood pressure, as measured at the brachial artery, and central systolic blood pressure, as estimated non-invasively by pulse wave analysis. We previously reported reference values for the central and peripheral pulse pressures and augmentation indexes by age in healthy Europeans (3). In the present study, we focused on the changes with age in central and peripheral systolic blood pressures, systolic amplification, and the difference between the augmented and non-augmented systolic blood pressures. In randomly recruited European subjects, we assessed these age-related changes cross-sectionally and in a subsample also longitudinally. We excluded patients on antihypertensive drug treatment from all analyses to avoid confounding by blood pressure lowering medications.

\section{Methods}

\section{Study population}

Recruitment for the Flemish Study on Environment, Genes and Health Outcomes (FLEMENGHO) started in 1985 (4,5). From August 1985 until November 1990, a random sample of the households living in a geographically defined area of Northern Belgium was investigated with the goal to recruit an equal number of participants in each of six subgroups by sex and age (20-39, 40-59 and $\geq 60$ years). All household members with a minimum age of 20 years were invited to take part, provided the quota of their sex-age group had not yet been fulfilled. From June 1996 until January 2004, recruitment of families continued using the former participants (1985 - 1990) as index persons and also including subjects younger than 20 years. The subjects were repeatedly followed up. In all study phases, i.e. at baseline and at follow-up, we employed the same standardized methods to measure peripheral and central blood pressure, laboratory tests and administer questionnaires.

The European Project on Genes in Hypertension (EPOGH) started in 1998 (6,7). The EPOGH investigators were trained at the Studies Coordinating Centre in Leuven, Belgium, and applied the same protocol, questionnaires and follow-up procedures, as used in FLEMENGHO. Questionnaires were translated from Dutch into Czech, Italian and Polish, and back-translated into Dutch to check that all questions had the same meaning in all languages. FLEMENGHO and EPOGH were conducted according to the principles outlined in the Helsinki Declaration for Investigation of Human Subjects (8). Each local Institutional Review Board approved the study protocol. Participants gave informed consent in writing.

As shown in Figure 1, 949 FLEMENGHO and 858 EPOGH participants had their blood pressure

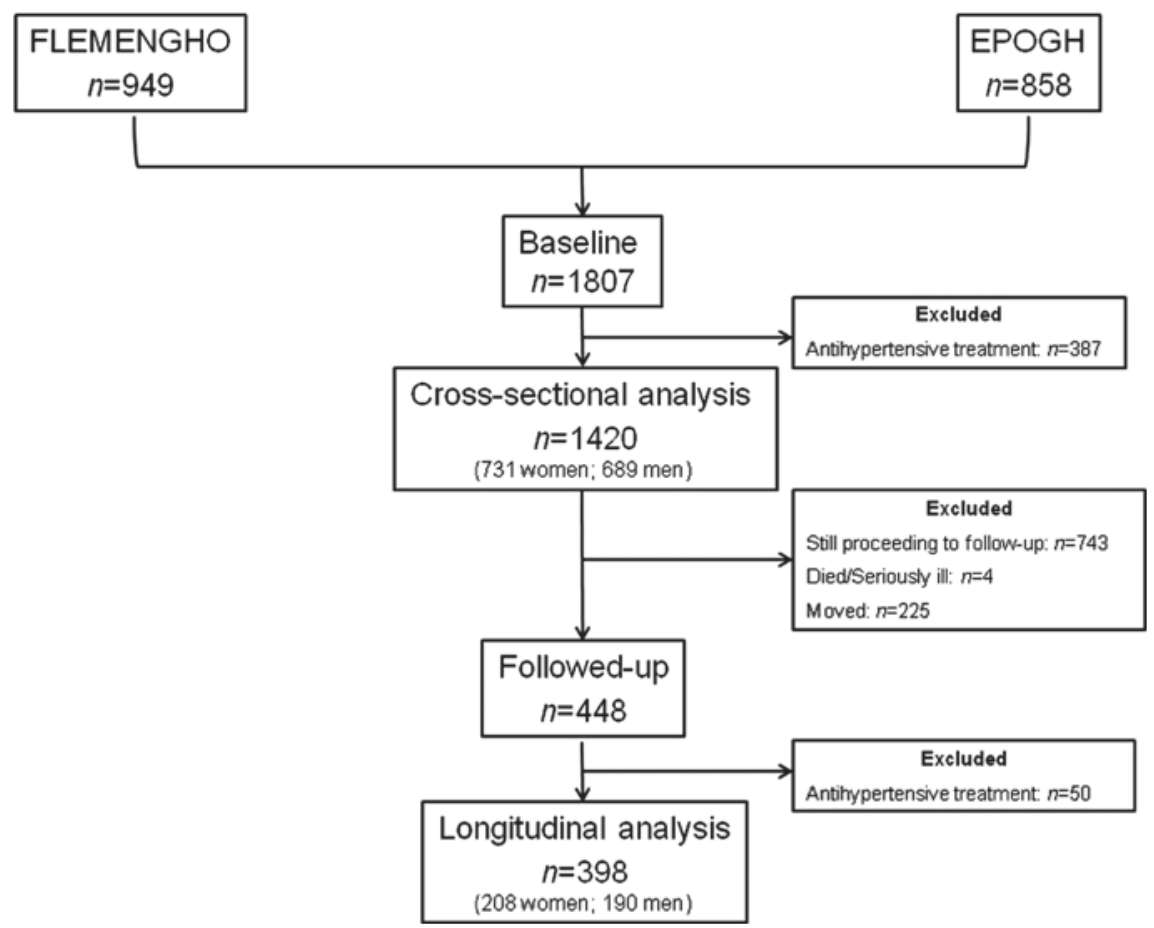

Figure 1. Flow chart of study participants. 
measured and their pulse wave analysed at baseline. Of 1807 participants with arterial measurements available, we excluded 387 on antihypertensive drug treatment, leaving 1420 subjects, 731 women and 689 men, for the cross-sectional analysis of baseline data. Of 1420 subjects included in the baseline analysis, three died, one was severely ill and 225 moved out of the study areas, and were therefore unavailable for the scheduled follow-up examination. Of the remaining 1191 subjects, 743 did not participate in the follow-up study because they are currently still proceeding to follow-up. We excluded from analysis an additional 50 subjects on antihypertensive drug treatment at follow-up. The number of subjects available for the longitudinal analyses totalled 398 (208 women and $190 \mathrm{men}$ ).

\section{Measurement of peripheral and central blood pressure}

The first phase of arterial phenotyping (baseline) took place from March 2002 until June 2009 in FLEMENGHO and from October 2000 until February 2008 in EPOGH. Arterial phenotyping at follow-up was done from February 2006 until June 2010 in FLEMENGHO and from January 2006 until July 2007 in the EPOGH centres.

To ensure steady state, measurements of peripheral and central blood pressures were obtained at local examination centres after the subjects had rested for at least $15 \mathrm{~min}$ in the supine position and had refrained from smoking, heavy exercise, and drinking alcohol or caffeinated beverages for at least $2 \mathrm{~h}$ prior to the examination. Brachial (peripheral) blood pressure was the average of three consecutive readings obtained by the validated (9) OMRON 705CP oscillometric sphygmomanometer (Omron Inc., Kyoto, Japan). For subjects with an arm circumference of less than $32 \mathrm{~cm}$, a standard cuff with an inflatable bladder of $22 \times 12 \mathrm{~cm}$ was used. For participants with a greater arm circumference, cuffs with a $35 \times 15 \mathrm{~cm}$ bladder were used. Hypertension was a blood pressure of at least $140 \mathrm{mmHg}$ systolic or $90 \mathrm{mmHg}$ diastolic.

Trained observers did the arterial measurements. During an 8-second period, they recorded the radial arterial waveform at the dominant arm by applanation tonometry. They used a high-fidelity SPC-301 micromanometer (Millar Instruments, Inc., Houston, TX, USA) interfaced with a laptop computer running the SphygmoCor software (AtCor Medical Pty. Ltd., West Ryde, New South Wales, Australia), version 6.31 at baseline and version 7.1 at follow-up. Recordings were discarded when the systolic or diastolic variability of consecutive waveforms exceeded $5 \%$ or when the amplitude of the pulse wave signal was less than $80 \mathrm{mV}$. The radial pulse wave was calibrated by the supine brachial blood pressure measured at the same arm, which as described above was measured immediately before the tonometric recordings. From the radial signal, the SphygmoCor software calculates the aortic pulse wave by means of a validated $(10,11)$ generalized transfer function. The software returns the central systolic blood pressure and the pressure at the first (P1) and second (P2) peak or shoulder of the central and radial waveforms.

In each country, the observers involved in the study took part in a reproducibility study of the SphygmoCor measurements. After repeat examination of 10-12 subjects, we computed the coefficient of variation as the ratio of the mean difference between repeat measurements to the standard deviation of the paired differences multiplied by 100 (12). Intra-observer and inter-observer variability were around $3 \%$ and $5 \%$, respectively (3).

For the present analysis, peripheral systolic blood pressure was the average of the three blood pressure readings obtained at the brachial artery for calibration of the pressure waves. Central systolic blood pressure was the maximum pressure of the central waveform. We calculated systolic augmentation by subtracting P1 from systolic blood pressure. Pressure amplification was defined as peripheral minus central systolic blood pressure.

\section{Other measurements}

We administered a standardized questionnaire to obtain information on each subject's medical history, smoking and drinking habits, and use of medications. Venous blood was collected after overnight fasting. We measured total serum cholesterol and blood glucose by automated enzymatic methods. Body mass index was body weight in kilograms divided by height in meters squared. Diabetes mellitus was defined as a fasting blood glucose concentration of at least 7.0 $\mathrm{mmol} / 1(126 \mathrm{mg} / \mathrm{dl})$ or the use of antidiabetic drugs.

\section{Statistical methods}

For database management and statistical analysis, we used SAS software, version 9.1.3 (SAS Institute, Cary, NC, USA). For comparison of means and proportions, we applied the large-sample $z$-test or $t$-test for paired observations and the $\chi^{2}$ statistic, respectively. For assessment of the cross-sectional association between blood pressure and age, we applied the PROC MIXED procedure, as implemented in the SAS software to account for family clusters. In the longitudinal analyses, we determined the significance of pressure amplification, systolic augmentation pressure and the longitudinal trends in the measurements by dividing the group mean differences or changes by the corresponding standard error (SE). In the last step of our analyses, we searched for variables associated with the peripheral and central blood pressure measurements, using stepwise linear regression. We 
set the $p$-values for variables to enter and to stay in the regression models at 0.05 . We tested the null hypothesis of no differences between the regression slopes of haemodynamic measurements on age, using multivariable analysis of variance as implemented in the MTEST statement of the PROC REG procedure of the SAS package (13). In all analyses, statistical significance was a $p$-value of 0.05 or less on twosided tests.

\section{Results}

\section{Characteristics of participants}

The 1420 participants included 731 women (51.5\%) and 278 (19.6\%) hypertensive patients, of whom by design of the analysis, none was taking antihypertensive drugs. Age ranged from 12-89 years. Table I gives the anthropometric characteristics, risk factors, and the peripheral and central haemodynamic measurements for women and men separately. Among all participants, fewer women than men smoked $(18.9 \%$ vs $29.7 \% ; p<0.0001)$ or reported regular alcohol consumption $(41.0 \%$ vs $71.7 \%$; $p<0.0001)$. Peripheral systolic, diastolic and pulse pressures were significantly lower in women than men $(p<0.0001)$. Similarly, central systolic and diastolic blood pressures $(p<0.0001)$, but not pulse pressure $(p=0.82)$, were lower in women.

Of 731 women and 689 men, $208(28.4 \%)$ and $190(27.6 \%)$, respectively, underwent a repeat examination at a median interval of 4.79 years (5th to 95th percentile interval, 3.96-5.98 years). Table I also provides the baseline characteristics of this subsample by sex. The characteristics of the subsample were similar to those of the whole study group.

\section{Associations of peripheral and central systolic blood pressures with age}

In cross-sectional analyses of 731 women and 689 men, the peripheral and central systolic blood pressures increased with age ( $p$ for trend $\leq 0.01$; Figure 2, Panels A and B). In single regression analysis, the cross-sectionally assessed age-related increase in central systolic blood pressure was larger than that in peripheral systolic pressure (Table II) both in women $(0.73$ vs $0.48 \mathrm{mmHg} /$ year; $p<0.0001)$ and in men (0.51 vs $0.27 \mathrm{mmHg} /$ year; $p<0.0001$ ). The slopes of peripheral and central systolic blood pressures on age were steeper $(p<0.001)$ in women than in men. The amplification pressure, the difference between peripheral and central systolic pressure decreased with age at a rate of $-0.24 \mathrm{mmHg} /$ year in both sexes

Table I. Baseline characteristics of participants by study phase and sex.

\begin{tabular}{|c|c|c|c|c|}
\hline \multirow[b]{2}{*}{ Characteristic } & \multicolumn{2}{|c|}{ All participants } & \multicolumn{2}{|c|}{ Participants with follow-up } \\
\hline & Women & Men & Women & Men \\
\hline Number & 731 & 689 & 208 & 190 \\
\hline Noorderkempen & $374(51.2)$ & $352(51.1)$ & $102(49.0)$ & $95(50.0)$ \\
\hline Pilsen & $73(10.0)$ & $73(10.6)$ & $43(20.7)$ & $27(14.2)$ \\
\hline Padova & $119(16.3)$ & $120(17.4)$ & - & - \\
\hline Kraków & $165(22.6)$ & $144(20.1)$ & $63(30.3)$ & $68(35.8)$ \\
\hline \multicolumn{5}{|l|}{ Anthropometrics } \\
\hline Age, year & $41.4 \pm 15.0$ & $42.0 \pm 15.7$ & $40.1 \pm 14.3$ & $39.2 \pm 15.7$ \\
\hline Height, cm & $163.4 \pm 6.5$ & $176.0 \pm 7.1^{\ddagger}$ & $164.4 \pm 6.9$ & $176.8 \pm 7.2^{\ddagger}$ \\
\hline Weight, kg & $66.0 \pm 12.8$ & $80.3 \pm 12.9^{\ddagger}$ & $65.8 \pm 12.6$ & $71.2 \pm 12.1^{\ddagger}$ \\
\hline Body mass index, $\mathrm{kg} / \mathrm{m}^{2}$ & $24.9 \pm 4.6$ & $25.9 \pm 4.1^{\ddagger}$ & $24.3 \pm 4.3$ & $25.3 \pm 3.5^{*}$ \\
\hline \multicolumn{5}{|l|}{ Risk factors } \\
\hline Current smoking, $n(\%)$ & $138(18.9)$ & $205(29.7)^{\ddagger}$ & $43(20.7)$ & $63(33.2)^{\dagger}$ \\
\hline Alcohol intake, $n(\%)$ & $300(41.0)$ & $494(71.7)^{\ddagger}$ & $59(28.4)$ & $105(55.3)^{\ddagger}$ \\
\hline Total cholesterol, mmol/1 & $5.05 \pm 1.05$ & $5.04 \pm 1.05$ & $5.13 \pm 1.10$ & $5.13 \pm 1.15$ \\
\hline Blood glucose, mmol/1 & $4.90 \pm 0.83$ & $5.17 \pm 1.20^{\ddagger}$ & $4.84 \pm 0.38$ & $5.20 \pm 0.47^{*}$ \\
\hline Hypertension, $n(\%)$ & $104(14.2)$ & $174(25.3)^{\ddagger}$ & $21(10.1)$ & $35(18.4)^{*}$ \\
\hline Diabetes, $n(\%)$ & $17(2.33)$ & $34(4.93)^{\dagger}$ & 0 & 0 \\
\hline Previous CVD, $n(\%)$ & $44(6.02)$ & $58(8.42)$ & $10(4.81)$ & $18(9.49)$ \\
\hline \multicolumn{5}{|l|}{ Peripheral haemodynamics } \\
\hline Systolic pressure, $\mathrm{mmHg}$ & $120.4 \pm 16.1$ & $127.6 \pm 14.9^{\ddagger}$ & $117.9 \pm 15.6$ & $124.6 \pm 12.6^{\ddagger}$ \\
\hline Diastolic pressure, $\mathrm{mmHg}$ & $75.1 \pm 10.2$ & $78.9 \pm 10.6^{\ddagger}$ & $73.8 \pm 9.7$ & $76.8 \pm 10.3^{\ddagger}$ \\
\hline Pulse pressure, $\mathrm{mmHg}$ & $45.3 \pm 11.3$ & $48.7 \pm 11.7^{\ddagger}$ & $44.1 \pm 10.5$ & $47.7 \pm 10.6^{\ddagger}$ \\
\hline Heart rate, beats/min & $66.8 \pm 10.2$ & $63.5 \pm 10.9^{\ddagger}$ & $67.3 \pm 10.1$ & $64.8 \pm 11.9^{*}$ \\
\hline \multicolumn{5}{|l|}{ Central haemodynamics } \\
\hline Systolic pressure, $\mathrm{mmHg}$ & $110.2 \pm 18.0$ & $113.7 \pm 15.4^{\ddagger}$ & $107.9 \pm 18.1$ & $109.8 \pm 13.3$ \\
\hline Diastolic pressure, $\mathrm{mmHg}$ & $76.1 \pm 10.4$ & $79.8 \pm 10.7^{\ddagger}$ & $74.8 \pm 9.8$ & $77.8 \pm 10.4^{\dagger}$ \\
\hline Pulse pressure, $\mathrm{mmHg}$ & $34.0 \pm 12.3$ & $33.9 \pm 10.2$ & $33.1 \pm 12.6$ & $32.0 \pm 8.9$ \\
\hline
\end{tabular}

Values are mean \pm SD or numbers of subjects (\%). -, missing information; CVD, cardiovascular disease. Hypertension was a blood pressure of at least $140 \mathrm{mmHg}$ systolic or $90 \mathrm{mmHg}$ diastolic. Significance of the sex difference ${ }^{*} p<0.05,{ }^{\dagger} p<0.01,{ }^{\ddagger} p<0.001$. 

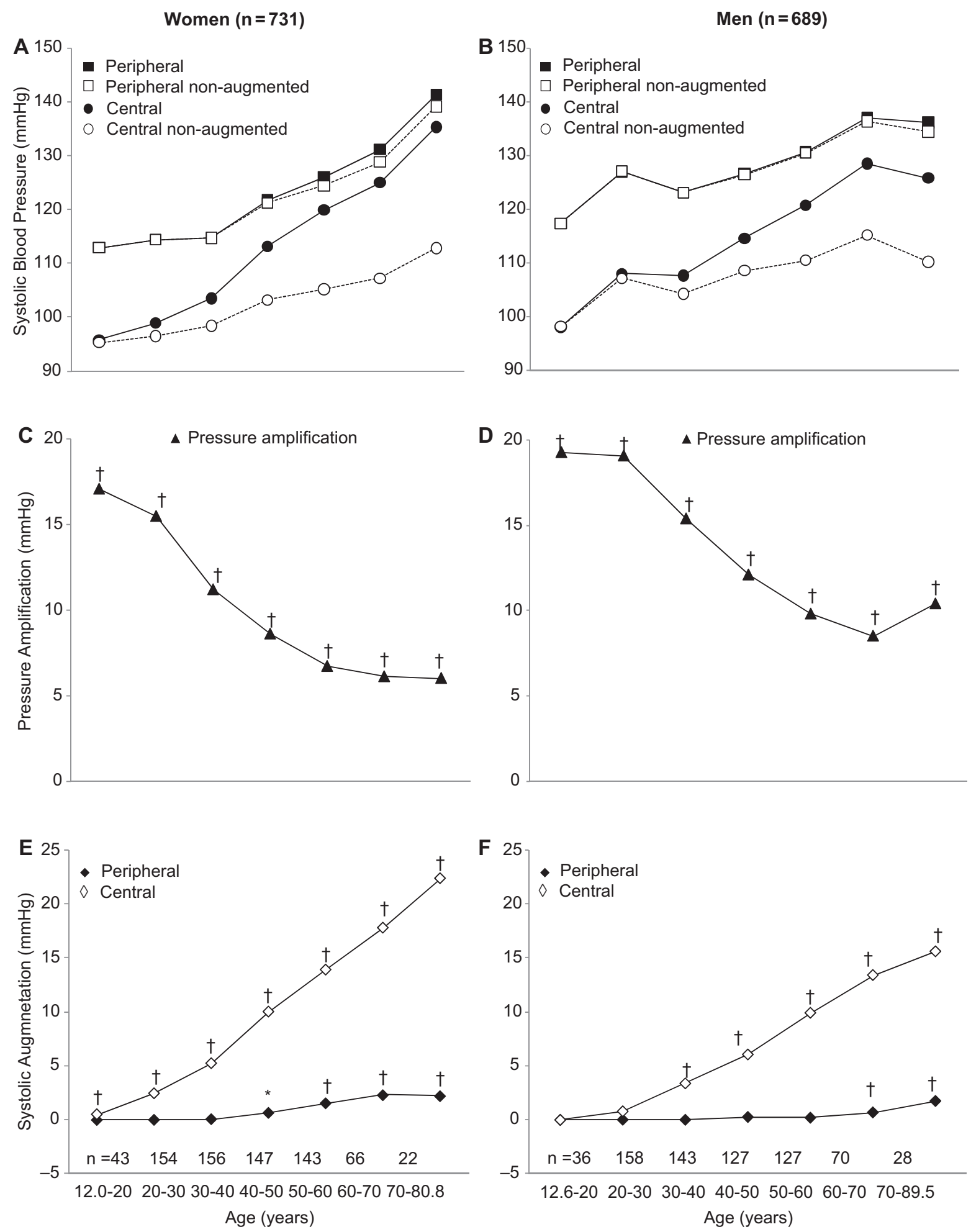

Figure 2. Association with age of peripheral and central systolic blood pressures (A, B), peripheral and central non-augmented systolic blood pressures (A, B), pressure amplification (C, D), and peripheral and central systolic augmentation (E, F) in women (A, C, E) and men (B, D, F). Peripheral systolic blood pressure was the average of three blood pressure readings at the brachial artery. Central systolic blood pressure was the maximum pressure of the central waveform. Systolic augmentation was obtained by subtracting the first systolic peak from systolic blood pressure. Pressure amplification is peripheral minus central systolic blood pressure. Plotted values are means for each age group. Numbers indicate the subjects contributing to the group means. All $p$-values for trend with age were statistically significant $(p<0.0001)$. Significance of the difference with zero: ${ }^{*} p<0.05,{ }^{\dagger} p<0.001$.

(Figure 2, Panels C and D and Table II; $p$-value for sex difference, 0.50).

Systolic augmentation pressure, the difference between maximal and non-augmented systolic pressure, increased with age, but the slope was smaller $(p<0.0001)$ for the peripheral than for the central augmentation pressure: 0.050 vs $0.395 \mathrm{mmHg}$ /year in women and 0.019 vs $0.301 \mathrm{mmHg} /$ year in men. The 
Table II. Cross-sectional association of blood pressure with age and longitudinal change in blood pressure with ageing in women and men.

\begin{tabular}{|c|c|c|c|c|}
\hline \multirow[b]{2}{*}{ Variables by sex Timing } & \multicolumn{3}{|c|}{ Cross-sectional associations with age $(\mathrm{mmHg} /$ year $)$} & \multirow{2}{*}{$\begin{array}{l}\text { Longitudinal change }(\mathrm{mmHg} / \text { year } \\
\text { Follow-up minus baseline }\end{array}$} \\
\hline & Baseline & Baseline & Follow-up & \\
\hline Women & All & Subgroup & Subgroup & Subgroup \\
\hline Number & 731 & 208 & 208 & 208 \\
\hline Peripheral systolic pressure & $0.48 \pm 0.03$ & $0.48 \pm 0.06$ & $0.58 \pm 0.07$ & $0.91 \pm 0.19$ \\
\hline Central systolic pressure & $0.73 \pm 0.03$ & $0.72 \pm 0.06$ & $0.79 \pm 0.06$ & $1.06 \pm 0.22$ \\
\hline Pressure amplification & $-0.24 \pm 0.01$ & $-0.23 \pm 0.02$ & $-0.22 \pm 0.02$ & $-0.27 \pm 0.07$ \\
\hline Peripheral systolic augmentation & $0.0500 \pm 0.0059$ & $0.0455 \pm 0.0084$ & $0.0955 \pm 0.0154^{*}$ & $0.1010 \pm 0.0267$ \\
\hline Central systolic augmentation & $0.395 \pm 0.013$ & $0.399 \pm 0.023$ & $0.472 \pm 0.026^{*}$ & $0.551 \pm 0.073$ \\
\hline \multicolumn{5}{|l|}{ Men } \\
\hline Number & 689 & 190 & 190 & 190 \\
\hline Peripheral systolic pressure & $0.27 \pm 0.03$ & $0.14 \pm 0.05$ & $0.21 \pm 0.06$ & $1.24 \pm 0.21$ \\
\hline Central systolic pressure & $0.51 \pm 0.03$ & $0.40 \pm 0.05$ & $0.49 \pm 0.06$ & $1.47 \pm 0.20$ \\
\hline Pressure amplification & $-0.24 \pm 0.01$ & $-0.26 \pm 0.02$ & $-0.29 \pm 0.02$ & $-0.23 \pm 0.09$ \\
\hline Peripheral systolic augmentation & $0.0187 \pm 0.0039$ & $0.0006 \pm 0.0002$ & $0.0167 \pm 0.0046^{*}$ & $0.0220 \pm 0.0104$ \\
\hline Central systolic augmentation & $0.301 \pm 0.012$ & $0.278 \pm 0.019$ & $0.330 \pm 0.023^{*}$ & $0.513 \pm 0.074$ \\
\hline
\end{tabular}

Regression coefficients $(\beta \pm \mathrm{SE}$ ) describe the cross-sectional associations of blood pressure with age. Subgroup refers to the subjects with repeat haemodynamic measurements at a median interval of 4.79 years. For the longitudinal assessment, values are average changes per year (follow-up minus baseline). Peripheral systolic blood pressure was the average of three blood pressure readings at the brachial artery. Central systolic blood pressure was the maximum pressure of the central waveform. Systolic augmentation was obtained by subtracting the first systolic peak from systolic blood pressure. Pressure amplification is peripheral minus central systolic blood pressure. All slopes on age were statistically significant $(p<0.01)$. In subjects with repeat examinations, an asterisk indicates a significant difference $(p \leq 0.039)$ between baseline and follow-up in the cross-sectionally assessed slopes on age. All changes from baseline to follow-up were significant $(p<0.05)$ except for peripheral systolic augmentation in men $(p=0.09)$.

slopes of peripheral and central systolic augmentation pressure were steeper $(p<0.001)$ in women than in men. In women ( $<20$ vs $40-50$ years) as well as in men (30-40 vs $60-70$ years), central systolic augmentation occurred at a younger age than peripheral systolic augmentation (Figure 2; Panels $\mathrm{E}$ and $\mathrm{F}$ ).

The cross-sectional findings in the subsample of 208 women and 190 men were generally consistent with those in the overall study population (Table II and Figure 3). The slopes on age of the peripheral and central systolic blood pressures and the amplification pressure were similar at baseline and follow-up $(P \geq 0.17)$. However, in the subsample, the crosssectionally assessed increase in the peripheral and central systolic augmentation pressures with age appeared to be smaller at baseline than at follow-up $(P \leq 0.01)$.

In the longitudinal analyses, all changes from baseline to follow-up were significant $(P \leq 0.039)$ except for the peripheral systolic augmentation in men $(p=0.09)$. The annual increases in the peripheral and central systolic blood pressures averaged $0.91 \mathrm{mmHg}$ and $1.06 \mathrm{mmHg}$ in women, and $1.24 \mathrm{mmHg}$ and $1.47 \mathrm{mmHg}$ in men (Table II and Figure 3$)$. The $p$-values for the sex differences were 0.12 and 0.08 , respectively. In the longitudinal analyses, women had more pressure amplification than men $(-0.27$ vs $-0.23 \mathrm{mmHg} /$ year; $p=0.001$; Table II). The annual changes in the peripheral and central systolic augmentation pressures were $0.10 \mathrm{mmHg}$ and $0.55 \mathrm{mmHg}$ in women, and 0.02 $\mathrm{mmHg}$ and $0.51 \mathrm{mmHg}$ in men. Longitudinally, the central systolic augmentation was similar in both sexes $(p=0.64)$, whereas the peripheral systolic augmentation was larger in women than in men $(p=0.002)$.

To evaluate to what extent regression-to-themean might influence the longitudinal changes in systolic blood pressure, we assessed these changes by quarters of the brachial (peripheral) systolic blood pressure at baseline (Table III). For peripheral, but not central systolic blood pressure, the increase over time weakened across the quarters in women. In men, the increases over time in both peripheral and central systolic blood pressure weakened across the quarters of baseline peripheral systolic blood pressure. In men, pressure amplification decline tended $(p=0.041)$ to increase with higher peripheral systolic blood pressure at baseline Table III).

\section{Multivariable analyses}

Table IV summarizes the results of stepwise regression in the cross-sectional analysis with peripheral and central systolic blood pressures as the dependent variables. Age contributed most of the explained variance. The multivariable-adjusted slopes of blood pressure on age were of similar magnitude as those in single regression analysis (Table II). In both women and men, the peripheral and central systolic blood pressures increased with body mass index and heart rate. The slopes of central blood pressure on age were significantly steeper than those of the peripheral systolic blood pressure. In women, the peripheral and central systolic blood pressure tended 

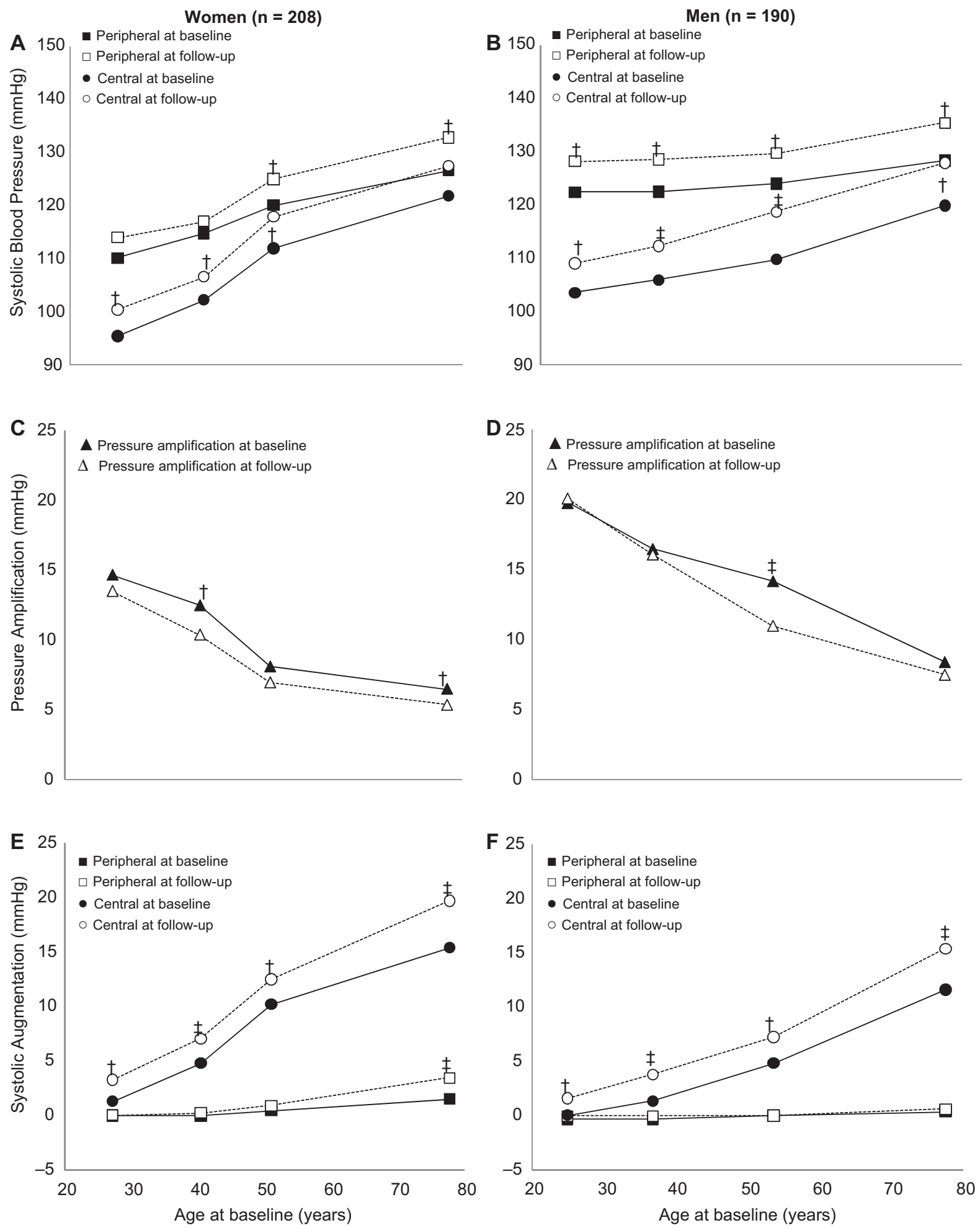

Figure 3. Blood pressures at baseline and follow-up by sex and quarters of the age distribution. Plotted values are peripheral and central systolic blood pressures (A, B), pressure amplification (C, D), and peripheral and central systolic augmentation (E, F) in 208 women (A, C, E) and 190 men $(B, D, F)$. All $p$-values for trend with age were statistically significant $(p<0.01)$. Significance of the difference between baseline and follow-up: ${ }^{*} p<0.05,{ }^{\dagger} p<0.01,{ }^{\ddagger} p<0.001$.

to be 2.81 and $2.31 \mathrm{mmHg}$ lower in smokers than non-smokers $(P \leq 0.08)$.

In the whole study group, pressure amplification significantly and independently decreased with age (partial regression coefficient $\pm S E$,
$-0.22 \pm 0.01 \mathrm{mmHg} /$ year; $p<0.0001)$, female sex $(-4.08 \pm 0.24 \mathrm{mmHg} ; p=0.0001)$ and current smoking $(-0.86 \pm 0.29 \mathrm{mmHg} ; p<0.003)$, whereas it increased with heart rate $(0.13 \pm 0.01 \mathrm{mmHg} /$ heart beat; $p<0.0001)$. 
Table III. Longitudinal changes in blood pressure by quarters of the distribution of peripheral systolic blood pressure at baseline in women and men.

\begin{tabular}{|c|c|c|c|c|c|}
\hline \multirow[b]{2}{*}{ Characteristic } & \multicolumn{4}{|c|}{ Quarters of the distribution of peripheral systolic blood pressure at baseline } & \multirow[b]{2}{*}{$p$-value } \\
\hline & Low & Medium-low & Medium-high & High & \\
\hline \multicolumn{6}{|l|}{ Women } \\
\hline Range of SBP at baseline, $\mathrm{mmHg}$ & $<108$ & $108-116$ & $117-126$ & $\geq 127$ & \\
\hline Number of subjects & 50 & 52 & 54 & 52 & \\
\hline \multicolumn{6}{|l|}{ Peripheral SBP } \\
\hline Baseline, mmHg & $101.3 \pm 4.1$ & $111.1 \pm 2.2$ & $118.8 \pm 2.3$ & $138.0 \pm 12.9$ & $<0.0001$ \\
\hline Follow-up, mmHg & $109.7 \pm 10.5$ & $117.4 \pm 10.1$ & $121.5 \pm 11.3$ & $137.5 \pm 17.4$ & $<0.0001$ \\
\hline Absolute change, $\mathrm{mmHg} /$ year & $1.73 \pm 2.36^{\ddagger}$ & $1.33 \pm 2.08^{\ddagger}$ & $0.82 \pm 2.45^{*}$ & $-0.13 \pm 3.51$ & 0.0028 \\
\hline \multicolumn{6}{|l|}{ Central SBP } \\
\hline Baseline, mmHg & $90.2 \pm 17.4$ & $100.8 \pm 5.4$ & $108.2 \pm 7.2$ & $128.2 \pm 13.7$ & $<0.0001$ \\
\hline Follow-up, mmHg & $100.5 \pm 10.1$ & $108.1 \pm 11.5$ & $113.2 \pm 12.6$ & $129.3 \pm 18.6$ & $<0.0001$ \\
\hline Absolute change, $\mathrm{mmHg} /$ year & $1.59 \pm 3.92^{\dagger}$ & $1.50 \pm 2.19^{\ddagger}$ & $1.08 \pm 2.59^{\dagger}$ & $0.18 \pm 3.50$ & 0.076 \\
\hline Pressure amplification, $\mathrm{mmHg}$ /year & $-0.34 \pm 1.16^{*}$ & $-0.17 \pm 1.04$ & $-0.26 \pm 0.76^{*}$ & $-0.31 \pm 0.81^{\dagger}$ & 0.50 \\
\hline \multicolumn{6}{|l|}{ Men } \\
\hline Range of SBP at baseline, $\mathrm{mmHg}$ & $<117$ & $117-124$ & $125-132$ & $\geq 133$ & \\
\hline Number of subjects & 45 & 49 & 48 & 48 & \\
\hline \multicolumn{6}{|l|}{ Peripheral SBP } \\
\hline Baseline, mmHg & $110.6 \pm 5.4$ & $119.7 \pm 1.5$ & $127.2 \pm 2.28$ & $141.1 \pm 9.6$ & $<0.0001$ \\
\hline Follow-up, mmHg & $122.8 \pm 13.6$ & $125.8 \pm 9.2$ & $130.7 \pm 12.1$ & $143.7 \pm 15.2$ & $<0.0001$ \\
\hline Absolute change, $\mathrm{mmHg} /$ year & $2.46 \pm 3.12^{\ddagger}$ & $1.29 \pm 1.97^{\ddagger}$ & $0.70 \pm 2.65$ & $0.49 \pm 3.40$ & 0.0043 \\
\hline \multicolumn{6}{|l|}{ Central SBP } \\
\hline Baseline, mmHg & $97.5 \pm 6.5$ & $105.5 \pm 5.6$ & $112.2 \pm 7.5$ & $124.4 \pm 13.7$ & $<0.0001$ \\
\hline Follow-up, mmHg & $109.9 \pm 12.8$ & $112.8 \pm 10.8$ & $117.0 \pm 13.0$ & $128.6 \pm 17.4$ & $<0.0001$ \\
\hline Absolute change, $\mathrm{mmHg} /$ year & $2.48 \pm 2.74^{\ddagger}$ & $1.56 \pm 1.85^{\ddagger}$ & $0.98 \pm 2.56^{*}$ & $0.85 \pm 3.54$ & 0.017 \\
\hline Pressure amplification, $\mathrm{mmHg}$ /year & $-0.02 \pm 1.46$ & $-0.26 \pm 0.98$ & $-0.28 \pm 1.35$ & $-0.36 \pm 1.16^{*}$ & 0.041 \\
\hline
\end{tabular}

Values are arithmetic means \pm SD. SBP, systolic blood pressure. All changes from baseline to follow-up were significant $(p<0.05)$. Change in blood pressure was computed by subtracting baseline from follow-up measurements. $p$-values are for trend across quarters. Significance of the within-group changes: ${ }^{*} p<0.05,{ }^{\dagger} p<0.01,{ }^{\ddagger} p<0.001$.

\section{Discussion}

The present reports focused on the changes with age in systolic augmentation of the central and peripheral systolic blood pressures in randomly recruited white Caucasians. In the cross-sectional analysis, the age-related increase was steeper for the central than for the peripheral systolic blood pressure. Systolic augmentation, defined as systolic blood pressure minus the first systolic peak, increased with age, but the increase was considerably larger for the central than for the peripheral systolic blood pressure. The amplification of systolic blood pressure, the difference between the peripheral systolic and central pressure therefore decreased with age.

Table IV. Correlates of peripheral and central systolic blood pressures in women and men.

\begin{tabular}{|c|c|c|c|c|c|c|}
\hline & \multicolumn{3}{|c|}{ Women $(n=731)$} & \multicolumn{3}{|c|}{ Men $(n=689)$} \\
\hline & Peripheral & Central & $p$ & Peripheral & Central & $p$ \\
\hline$R^{2}$ & 0.28 & 0.40 & & 0.14 & 0.29 & \\
\hline Intercept & 69.4 & 57.1 & & 95.9 & 72.5 & \\
\hline \multicolumn{7}{|l|}{ Age (years) } \\
\hline$\beta \pm S E$ & $0.421 \pm 0.038^{\Im}$ & $0.651 \pm 0.039 \S$ & $<0.0001$ & $0.236 \pm 0.037^{\S}$ & $0.464 \pm 0.035^{\S}$ & $<0.0001$ \\
\hline Partial $r^{2}$ & 0.198 & 0.359 & & 0.076 & 0.261 & \\
\hline \multicolumn{7}{|c|}{ Body mass index, $\mathrm{kg} / \mathrm{m}^{2}$} \\
\hline$\beta \pm S E$ & $0.830 \pm 0.120^{\S}$ & $0.815 \pm 0.122^{\Im}$ & 0.546 & $0.486 \pm 0.146^{\ddagger}$ & $0.499 \pm 0.137^{\S}$ & 0.474 \\
\hline Partial $r^{2}$ & 0.062 & 0.043 & & 0.014 & 0.017 & \\
\hline \multicolumn{7}{|c|}{ Heart rate, beats/min } \\
\hline$\beta \pm S E$ & $0.202 \pm 0.051^{\dagger}$ & $0.094 \pm 0.053^{*}$ & 0.172 & $0.287 \pm 0.049^{\ddagger}$ & $0.136 \pm 0.046^{\ddagger}$ & 0.109 \\
\hline Partial $r^{2}$ & 0.016 & 0.003 & & 0.052 & 0.009 & \\
\hline \multicolumn{7}{|c|}{ Smoking $(0,1)$} \\
\hline$\beta \pm S E$ & $-2.811 \pm 1.308^{\dagger}$ & $-2.311 \pm 1.334^{*}$ & 0.182 & ns & ns & - \\
\hline Partial $r^{2}$ & 0.005 & 0.002 & & & & \\
\hline
\end{tabular}

Values are partial regression coefficients $(\beta \pm \mathrm{SE})$ or partial coefficients of determination $\left(r^{2}\right)$. Significance of the partial regression coefficient: ns $p \geq 0.10 ;{ }^{*} p<0.10 ;{ }^{\dagger} p<0.05,{ }^{\ddagger} p<0.01,{ }_{p} p<0.001$. The covariables considered in the stepwise regression procedure were: age, body mass index, heart rate and current smoking and drinking. $p$-values are for the comparison of the partial regression coefficients describing the associations with peripheral vs central blood pressure. -, not applicable. 
Our cross-sectional observations are in agreement with known physiological concepts, initially tested in experimental studies (14) and in selected patients (14-16). They are also in line with several previously published cross-sectional population studies (17-19), including the Anglo-Cardiff Collaborative Trial (ACCT) (17). McEniery and colleagues (17) studied 4001 healthy, normotensive individuals, aged 18-90 years. In both women and men, central systolic pressure increased more with age than did peripheral systolic blood pressure $(p<0.001)$. As in our current cross-sectional analyses, the increase in central systolic pressure was more prominent in women than men $(p=0.01)$. Wilkinson and coworkers (18) combined volunteers recruited from the community and patients attending an open access clinic for the assessment of cardiovascular risk. They excluded patients with a cardiovascular disease, diabetes or on drug treatment, but not those with hypertension. Pressure amplification, defined as the peripheral-tocentral pulse pressure ratio decreased linearly with age $(r=-0.7 ; p<0.001)$. However, the relation with age of the ratio of peripheral pulse pressure to the non-augmented central pulse pressure was not statistically significant $(r=0.1 ; p=0.1)$.

In a cross-sectional analysis (19), Mitchell and coworkers measured proximal aortic pressure and flow, forward pressure wave amplitude, global wave reflection, reflected wave timing, and pulse wave velocity non-invasively in 6417 participants of the Framingham Heart Study (age range 19-90 years; $53 \%$ women). Across the age range, the forward wave amplitude explained most of the variance of central $(89 \%)$ and peripheral $(84 \%)$ pulse pressures. Below 50 years of age, the central and peripheral pulse pressures decreased with age, but reflected waves increased with advancing age, whereas the opposite occurred above age 50 years. The forward wave amplitude explained $80 \%$ (central) and $66 \%$ (peripheral) of the variance in pulse pressure in younger participants ( $<50$ years) and $90 \%$ and $84 \%$ in the older participants $(\geq 50$ years; all $p<0.0001$ ) (19).

A recent publication of the ACCT (20) involved healthy people, subjects with risk factors, such as hypertension, hypercholesterolaemia, smoking, diabetes, diabetic patients and patients with a history of cardiovascular disease. The 10,613 participants were 18-101 years old (50.8\% women). In this report, McEniery and colleagues chose to express aortic pulse pressure relative to brachial pulse pressure, i.e. the reciprocal of the traditionally used pressure amplification. Higher values of this new index represent subjects with a relatively higher aortic pressure for a given brachial pressure. The aorta-to-brachial pulse pressure ratio averaged 0.72 in healthy subjects and was consistently and significantly higher in all other groups (range of means $0.77-0.80$ ) with the exception of smokers (mean value $=0.66$ ).
In our current study, peripheral and central systolic blood pressures were lower in smoking than non-smoking women. Smoking acutely raises blood pressure through sympathetic stimulation (21), and is associated with higher blood pressure, at least if the latter is continuously recorded (22). In contrast, in epidemiological studies in which the conventional blood pressure was measured after a smoking-free interval, smokers show slightly lower blood pressure levels than non-smokers (23). This may be related to the reduction in sympathetic activity in the intervals between smoking (24), the development of tolerance (25) or to the lower body weight of smokers. In McEniery's report, ageing was associated with an increased aorta-to-brachial pulse pressure ratio. Above 80 years, the difference between peripheral and central systolic blood pressure still averaged $8 \mathrm{mmHg}$ in women and $11 \mathrm{mmHg}$ in men. In our current study, the corresponding values were $6.0 \mathrm{mmHg}(n=22)$ and $10.4 \mathrm{mmHg}(n=28)$, respectively.

To our knowledge, our current study is among the first to examine pressure amplification in a longitudinal manner. The age-related increases in peripheral and central systolic blood pressures were consistently greater on longitudinal compared with the cross-sectional assessment. Because of the stringent quality control programme set up for the blood pressure measurements (6), it is unlikely that drift in methodology can explain these observations. Calculating a difference between repeated measurements in the same individual might not be equivalent to computing a correlation coefficient across different subjects. As shown in Table III, longitudinal data are sensitive to regression-to-the-mean. Furthermore, systolic blood pressure is a major risk factor. Subjects at the higher end of the distribution of systolic blood pressure are more likely to experience cardiovascular complications or die, and therefore to disappear from follow-up. The unmeasured attrition in the population available for cross-sectional analysis might at least contribute to the larger estimates of the agerelated increase in the longitudinal assessment. Women have a longer life expectancy than men. Stronger attrition among men might explain why in the longitudinal analysis pressure amplification decreased more over time in women than men $(-0.27$ vs $-0.23 \mathrm{mmHg} /$ year), whereas in the crosssectional analysis there was no sex difference $(-0.24$ vs $-0.24 \mathrm{mmHg} /$ year). Because central systolic blood pressure is a predictor of cardiovascular complications (26-30), the latter assumptions need to be ascertained in other prospective population studies.

The present study should be interpreted within the context of its potential limitations. First, in contrast to other studies on the age dependency of arterial stiffness $(17,18,31,32)$, we did not exclude patients with hypertension, diabetes or previous cardiovascular disease. On the other hand, we excluded patients on antihypertensive drug treatment. Second, in our current 
study, as in all other cross-sectional studies, age was the overriding determinant of the peripheral and central systolic blood pressures with smaller contributions of sex, body mass index, heart rate and smoking. Our sample size might have been insufficient to identify other blood pressure correlates, such as alcohol intake, serum total cholesterol or blood glucose. Finally we did not assess the pulse wave contour at the brachial artery and we could therefore not account for any brachial-to-radial amplification, which as estimated non-invasively but not invasively contributes to total amplification and increase with age $(33,34)$. However, brachial tonometry is technically demanding $(34,35)$. In experienced hands, it is successful in less than $80 \%$ of individuals (35).

Our current findings set an agenda for research. Indeed, they are based on white Caucasian populations with a western life style, high prevalence of obesity and relatively high cholesterol levels. They cannot be extrapolated to other ethnicities or populations with different lifestyles. It would be of great interest to explore whether our current findings can be replicated in lean Asian people, who generally have lower serum cholesterol levels. The hypothesis of an underestimation of the association between systolic blood pressure and age in cross-sectional studies needs clarification. Finally, the currently available literature on the amplification of systolic blood pressure is difficult to interpret, because varying definitions are being used $(19,20)$. Experts committees might wish to provide guidance in standardizing the terminology.

\section{Acknowledgment}

The authors gratefully acknowledge expert assistance of Ms Sandra Covens and Ms Ya Zhu (University of Leuven).

\section{Sources of funding}

The European Union (grants IC15-CT98-0329EPOGH, LSHM-CT-2006-037093 InGenious HyperCare, and HEALTH-F4-2007-201550 Hypergenes) supported the Studies Coordinating Centre (Leuven, Belgium) and the studies in Pilsen (Czech Republic), Padova (Italy), Kraków (Poland) and Novosibirsk (Russian Federation). The Studies Coordinating Centre also received grants from the Fonds voor Wetenschappelijk OnderzoekVlaanderen, Ministry of the Flemish Community, Brussels, Belgium (grants G.0575.06 and G.0734.09), and the Katholieke Universiteit Leuven, Belgium (grants OT/00/25 and OT/05/49).

Declaration of interest: The authors report no conflicts of interest. The authors alone are responsible for the content and writing of the paper.

\section{References}

1. Staessen J, Amery A, Fagard R. Editorial review. Isolated systolic hypertension in the elderly. J Hypertens. 1990;8: 393-405.

2. O'Rourke MF, Kelly PJ. Wave reflections in the systemic circulation and its implications in ventricular function. J Hypertens. 1993;11:327-337.

3. Wojciechowska W, Staessen JA, Nawrot T, Cwynar M, Sleidlerová J, Stolarz K, et al. Reference values in White Europeans for the arterial pulse wave recorded by means of the ShygmoCor device. Hypertens Res. 2006;29:475-483.

4. Staessen JA, Wang JG, Brand E, Barlassina C, Birkenhager WH, Hermann SM, et al. Effects of three candidate genes on prevalence and incidence of hypertension in a Caucasian population J Hypertens. 2001;19:1349-1358.

5. Li Y, Zagato L, Kuznetsova T, Tripodi G, Zerbini G, Richart $\mathrm{T}$, et al. Angiotensin-converting enzyme I/D and $\alpha$-adducin Gly460Trp polymorphisms. From angiotensin-converting enzyme activity to cardiovascular outcome. Hypertension. 2007;49:1291-1297.

6. Kuznetsova T, Staessen JA, Kawecka-Jaszcz K, Babeanu S, Casiglia E, Filipovský J, et al. Quality control of the blood pressure phenotype in the European Project on Genes in Hypertension. Blood Press Monit. 2002;7:215-224.

7. Wojciechowska W, Staessen JA, Stolarz K, Nawrot T, Filipovský J, Tichá $M$, et al. Association of peripheral and central arterial wave reflections with the CYP11B2 -344C allele and sodium excretion. J Hypertens. 2004;22:2311-2319.

8. 41st World Medical Assembly. Declaration of Helsinki: Recommendations guiding physicians in biomedical research involving human subjects. Bull Pan Am Health Organ. 1990; 24:606-609.

9. O'Brien E, Mee F, Atkins N, Thomas M. Evaluation of three devices for self-measurement of blood pressure according to the revised British Hypertension Society Protocol: The Omron HEM-705 CP, Philips HP5332 and Nissei DS-175. Blood Press Monit. 1996;1:55-62.

10. Karamanoglu M, O'Rourke MF, Avolio AP, Kelly PJ. An analysis of the relationship between central aortic and peripheral upper limb pressure waves in man. Eur Heart J. 1993;14: 160-167.

11. Pauca AL, O'Rourke M, Kon ND. Prospective evaluation of a method for estimating ascending aortic pressure from the radial artery pressure waveform Hypertension. 2001;38: 932-937.

12. Bland JM, Altman DG. Statistical methods for assessing agreement between two methods of clinical measurement. Lancet. 1986;1:307-310.

13. Staessen J, O'Brien E, Atkins N, Bulpitt CJ, Cox J, Fagard R, et al. The increase in blood pressure with age and body mass index is overestimated by conventional sphygmomanometry. Am J Epidemiol. 1992;136:450-459.

14. O'Rourke M. Arterial haemodynamics and ventricular-vascular interaction in hypertension. Blood Press. 1994;3:33-37.

15. Murgo JP, Westerhof N, Giolma JP, Altobelli SA. Aortic input impedance in normal man: Relationship to pressure wave forms. Circulation. 1980;62:105-116.

16. Wilkinson IB, MacCallum H, Flint L, Cockroft JR, Newby $\mathrm{DE}$, Webb DJ. The influence of heart rate on augmentation index and central arterial pressure in humans. J Physiol. 2000; 525:263-270.

17. McEniery CM, Yasmin, Hall IR, Qasem A, Wilkinson IB, Cockroft JR, et al. Normal vascular ageing: Differential effects on wave reflection and aortic pulse wave velocity. The AngloCardiff Collaborative Trial (ACCT). J Am Coll Cardiol. 2005; 46:1753-1760.

18. Wilkinson IB, Franklin SS, Hall IR, Tyrrell S, Cockcroft JR. Pressure amplification explains why pulse pressure is unrelated to risk in young subjects. Hypertension. 2001;38: 1461-1466. 
19. Mitchell GF, Wang N, Palmisano JN, Larson MG, Hamburg NM, Vita JA, et al. Hemodynamic correlates of blood pressure across the adult age spectrum: Noninvasive evaluation in the Framingham Heart Study. Circulation. 2010;122: 1379-1386.

20. McEniery CM, Yasmin, McDonnell B, Munnery M, Wallace SM, Rowe CV, et al. Central pressure: Variability and impact of cardiovascular risk factors. The Anglo-Cardiff collaborative trial II. Hypertension. 2008;51:1-7.

21. Cryer PE, Haymond MW, Santiago JV, Shah SD. Norepinephrine and epinephrine release and adrenergic mediation of smoking-associated hemodynamic and metabolic events. $\mathrm{N}$ Engl J Med. 1976;295:573-577.

22. Groppelli A, Omboni S, Parati G, Mancia G. Blood pressure and heart rate response to repeated smoking before and after $\beta$-blockade and $\alpha_{1}$-inhibition. J Hypertens. 1990;8 Suppl 5:S35-S40

23. Benowitz NL, Sharp DS. Inverse relation between serum cotinine concentration and blood pressure in cigarette smokers. Circulation. 1989;80:1309-1312.

24. St George IM, Williams S, Stanton WR, Silva PA. Smoking and blood pressure in 15 year olds in Dunedin, New Zealand. Brit Med J. 1991;302:89-90.

25. Benowitz NL. Pharmacologic aspects of cigarette smoking and nicotine addiction. N Engl J Med. 1988;319:1318-1330.

26. Laurent S, Boutouyrie P, Asmar R, Gautier I, Laloux B, Guize $\mathrm{L}$, et al. Aortic stiffness is an independent predictor of allcause and cardiovascular mortality in hypertensive patients. Hypertension. 2001;37:1236-1241.

27. Safar ME, Blacher J, Pannier B, Guerin AP, Marchais SJ, Guyonvarc'h PM, et al. Central pulse pressure and mortality in end-stage renal disease. Hypertension. 2002;39: 735-738.

28. Vlachopoulos C, Aznaouridis K, Stefanadis C. Prediction of cardiovascular events and all-cause mortality with arterial stiffness: A systematic review and meta-analysis. J Am Coll Cardiol. 2010;55:1318-1327.

29. Benetos A, Thomas F, Joly L, Blacher J, Pannier B, Labat C, et al. Pulse pressure amplification. A mechanical biomarker of cardiovascular risk. J Am Coll Cardiol. 2010;55:1032-1037.

30. Jankowski P, Kawecka-Jaszcz K, Czarnecka D, BrzozowskaKiszka M, Styczkiewicz K, Loster M, et al. Pulsatile but not steady component of blood pressure predicts cardiovascular events in coronary patients Hypertension. 2008;51:848-855.

31. Mitchell GF, Parise H, Benjamin EJ, Larson MG, Keyes MJ, Vita JA, et al. Changes in arterial stiffness and wave reflection with advancing age in healthy men and women. The Framingham Heart Study. Hypertension. 2004;43:1239-1245.

32. Li Y, Staessen JA, Li LH, Huang QF, Lu L, Wang JG. Reference values for the arterial pulse wave in Chinese. Am J Hypertens. 2008;21:668-673.

33. Segers P, Mahieu D, Kips J, Rietzschel E, De Buyzere M, De Bacquer D, et al. Amplification of the pressure pulse in the upper limb in healthy, middle-aged men and women. Hypertension. 2009;54:414-420.

34. O'Rourke MF, Adji A. Clinical use of applanation tonometry: Hope remains in Pandora's box J Hypertens. 2010;28:229-233.

35. Segers P, Rietzschel ER, De Buyzere ML, Vermeersch SJ, De Bacquer D, Van Bortel LM, et al. Noninvasive (input) impedance, pulse wave velocity, and wave reflection in healthy middleaged men and women. Hypertension. 2007;49:1248-1255. 